\title{
Polar properties and local piezoelectric response of ferroelectric nanotubes
}

\author{
A.N. Morozovska ${ }^{1 *}$, G.S. Svechnikov ${ }^{1}$, E.I. Shishkin ${ }^{2}$, V.Y. Shur ${ }^{2}$ \\ ${ }^{I}$ V. Lashkaryov Institute of Semiconductor Physics, National Academy of Science of Ukraine \\ 41, prospect Nauky, 03028 Kyiv, Ukraine \\ ${ }^{2}$ Institute of Physics and Applied Mathematics, Ural State University, 620083 Ekaterinburg, Russia \\ *Corresponding author e-mail:morozo@i.com.ua
}

\begin{abstract}
We consider the polar properties of the ferroelectric nanotubes within the framework of Landau-Ginzburg-Devonshire phenomenology. The approximate analytical expression for the paraelectric-ferroelectric transition temperature dependence on the radii of nanotube, polarization gradient, extrapolation length, elastic stresses and strains arising from surface tension and thermal expansion mismatch, and electrostriction coefficient was derived. We calculated effective local piezoresponse of the ferroelectric nanotube within decoupling approximation of electric and elastic problem. Obtained results explain the ferroelectricity conservation in $\mathrm{Pb}(\mathrm{Zr}, \mathrm{Ti}) \mathrm{O}_{3}$ and $\mathrm{BaTiO}_{3}$ nanotubes observed by using Piezoelectric Force Microscopy.
\end{abstract}

Keywords: polar properties, ferroelectric nanotube, effective local piezoresponse, piezoelectric force microscopy.

Manuscript received 18.09.08; accepted for publication 20.10.08; published online 11.11.08.

\section{Introduction}

Ferroelectric nanotubes and nanorods are actively studied in nano-physics and nano-technology [1-5]. In many cases they demonstrate such polar properties as remnant polarization [1] and local piezoelectric hysteresis [3-5].

It is generally accepted, that the ferroelectric properties disappear under the particle size decreases below the critical one [6]. Actually, it is well known that depolarization electric field exists in the majority of confined ferroelectric systems [7] and causes the sizeinduced ferroelectricity disappearance in thin films and spherical particles [8].

However, the cylindrical geometry does not destroy ferroelectric phase (in contrast to size-induced paraelectric phase in spherical particles [9-15]), but sometimes the noticeable enhancement of ferroelectric properties appears $[1-5,16]$. For instance, Yadlovker and Berger [1] reported about the spontaneous polarization enhancement up to $0.25-2 \mu \mathrm{C} / \mathrm{cm}^{2}$ and ferroelectric phase conservation in Rochelle salt nanorods. With the help of Piezoelectric Force Microscopy (PFM), Morrison et al. $[4,5]$ demonstrated that $\mathrm{PbZr}_{0.52} \mathrm{Ti}_{0.48} \mathrm{O}_{3}$ (PZT) nanotubes (radius $R=500-700 \mathrm{~nm}$, thickness $h=50-70 \mathrm{~nm}$, length $50 \mu \mathrm{m}$ ) possesses rectangular shape of the local piezoelectric response hysteresis loop with effective remnant piezoelectric coefficient value compatible with the ones typical for PZT films. Also, the authors demonstrated that the ferroelectric properties of the free $\mathrm{BaTiO}_{3}$ nanotubes are perfect. Poyato et al. [17] with the help of PFM found that nanotube-patterned ("honeycomb") $\mathrm{BaTiO}_{3}$ film of thickness 200-300 nm reveal ferroelectric properties. The inner diameter of the nanotubes ranged from 50 to $100 \mathrm{~nm}$. Also, they demonstrated the existence of local piezoelectric and oriented ferroelectric responses, prior to the application of a dc field, in nanotubes-patterned $\mathrm{BaTiO}_{3}$ thin films on Ti substrates synthesized hydrothermally at $200{ }^{\circ} \mathrm{C}$. The phenomenological description of ferroelectricity enhancement in cylindrical nanoparticles has been recently proposed [18-20].

In our consideration of ferroelectric nanotube, we suppose that a nanoparticle surface is covered with a charged layer consisted of the free carriers adsorbed from the ambient (e.g., air with definite humidity or pores filled with a precursor solution). For instance, a thin water layer condensates on the polar oxide surface in the air with humidity $20-50 \%\left[{ }^{2} 1\right]$. The surface charges screen the surrounding template (usually $\mathrm{Si}$ or alumina porous matrix $[1,16]$ or regular $2 \mathrm{D}$ photonic crystal [3-5]) from the nanoparticle electric field, but the depolarization field inside the particle is caused by inhomogeneous polarization distribution. Thus, one could calculate the depolarization field inside a cylindrical nanoparticle under the short-circuit conditions proposed by Kretschmer and Binder [22]. 
For the case when a liquid precursor of ferroelectric (e.g. RS, PZT, SBT or BTO) filled the porous template by capillary effects [1], the uniform stress inside the pores is caused by surface tension [18]. During the following annealing both the thermal stresses and misfit strain on the tube-pore interface usually appear. In most of cases the stress causes the thin strained layer ("shell") on the tube-pore interface. For instance, Luo et al. [3] and Morrison et al. [4, 5] reported about amorphous PZT layer of thickness $\Delta h \approx 5-20 \mathrm{~nm}$ that clamped the nanotube crystalline "core". The shell may be partially removed by selective etching.

Using the experimental background, we modified the core-and-shell model of spherical ferroelectric nanoparticles proposed earlier by Niepce et al. [23, 24] and Glinchuk et al. [25] for the description of ferroelectric nanotubes polar properties. Firstly, we obtained the solution of elastic problem for the stress distribution inside the tube covered with thin strained shell. Then the polarization distribution inside the core was obtained and approximate analytical expression for paraelectric-ferroelectric transition temperature dependence on the nanotube thickness and radius, effective stress, polarization gradient and electrostriction coupling coefficients was derived. Note that the stress is caused by the particle surface clamping by template, i.e. it is related to surface tension, thermal expansion and mismatch strain [26]. We proved that the reason of the polar properties enhancement and conservation in ferroelectric nanotubes is the stress coupled with polarization via electrostriction effect under the strong decrease of depolarization field with tube length increase. Within decoupling approximation of electric and elastic problem $[27,28]$, we calculated the tube PFM response and compared calculations with available experimental data.

\section{Free energy of a nanotube in the core and shell model}

Let us consider the ferroelectric cylindrical nanotube of outer radius $R_{o}$, inner radius $R_{i}$, thickness $h=R_{o}-R_{i}$ and height $l$ (see Fig. 1). The tube "core" of thickness $h-\Delta h$ is covered with thin amorphous "shell" of thickness $\Delta h<<h$. The core polarization $P_{3}$ is oriented along $z$ axes. The external electric field is $\mathbf{E}=\left(0,0, E_{0}\right)$.

For the case when a liquid precursor of ferroelectric filled the porous template by capillary effects, the uniform stress $\sigma_{i j}=-p_{o} \delta_{i j}$ is caused by surface tension $p_{o}=2 \mu / R_{o}$ [18], where the coefficient $\mu$ could be related to the effective surface tension. During the following thermal annealing the cubic structured or even amorphous shell layer of thickness $\Delta h<<R_{o}$ appears at the nanotube-template interface. The nanotube crystalline core is strained allowing for the thermal expansion and growth deformations as well as the surface tension frozen up inside the shell. Even after the
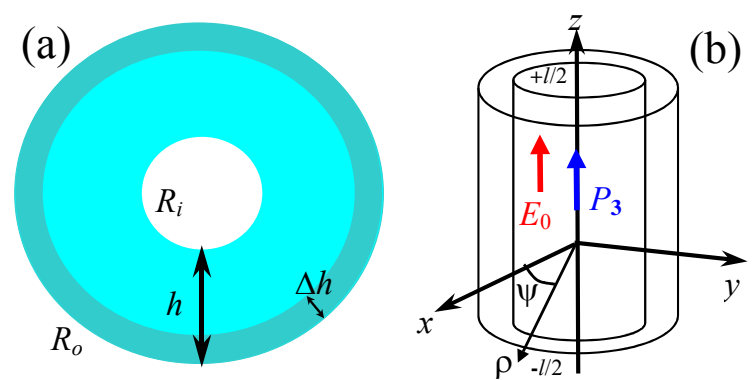

Fig. 1. (a) Cross-section of the ferroelectric nanotube with "shell" of thickness $\Delta h$ and "core" of thickness $h-\Delta h$. (b) Geometry of calculations in cylindrical coordinates $\{\rho, \psi, z\}$.

template removal, the internal strain $u_{i j}=u_{t} \delta_{i j}$ is different inside core and shell. Namely, the shell strain $u_{t}^{s}$ is determined by the effective surface tension $p_{o}$, growth deformation $u_{g}$ and thermal expansion during annealing at temperature $T_{a}$, i.e. $u_{t}^{s}=-p_{o}\left(s_{11}+2 s_{12}\right)+\vartheta_{i}^{s} \Delta T+u_{g}$, whereas the core strain is determined by the thermal expansion only, i.e. $u_{t}^{c}=\vartheta_{i}^{c} \Delta T$ (here $s_{11}$ and $s_{12}$ are the elastic compliances, $\vartheta_{i}^{c}$ and $\vartheta_{i}^{s}$ are the linear temperature expansion coefficients of the tube core and shell, $\Delta T=T-T_{a}$ is the temperature change, see e.g. $[29,30])$. The difference $\Delta u=u_{t}^{c}-u_{t}^{s}$ determines the core stress $\sigma_{i j}^{c}$ as:

$\sigma_{11}^{c}+\sigma_{22}^{c} \approx \sigma_{33}^{c} \approx \sigma\left(R_{o}, R_{i}\right)$,

$\sigma\left(R_{o}, R_{i}\right)=-\frac{\Delta u}{s_{11}+s_{12}} \frac{2 R_{o} \Delta h}{\left(R_{o}+R_{i}\right) h}$,

$\Delta u=\frac{2 \mu}{R_{o}}\left(s_{11}+2 s_{12}\right)+\left(\vartheta_{i}^{c}-\vartheta_{i}^{s}\right) \Delta T-u_{g}$.

The Euler-Lagrange equation for the polarization can be obtained by the variation on polarization of the free energy functional $G=G_{V}+G_{S}$, consisting of the bulk part $G_{V}$ and the surface one $G_{S}$. The bulk part $G_{V}$ acquires the form:

$$
\begin{aligned}
& G_{V}=\int_{-l / 2}^{l / 2} d z \int_{0}^{2 \pi} d \psi \int_{R_{i}}^{R_{o}-\Delta h} \rho d \rho \times\left(\frac{\alpha_{R}(T)}{2} P_{3}^{2}+\right. \\
& \left.+\frac{\beta_{R}}{4} P_{3}^{4}+\frac{\gamma}{6} P_{3}^{6}+\frac{\delta}{2}\left(\nabla P_{3}\right)^{2}-P_{3}\left(E_{0}+\frac{E_{3}^{d}}{2}\right)\right) .
\end{aligned}
$$

Material coefficients $\delta>0$ and $\gamma>0$, coefficient $\beta<0$ for the first order phase transitions or $\beta>0$ for the second order ones. The depolarization field is denoted by $E_{3}^{d}$. The coefficients $\alpha_{T}(T)$ and $\beta_{R}$ is renormalized by elastic stresses $\sigma_{i i}^{c}$ as 


$$
\begin{aligned}
& \alpha_{R}\left(T, R_{o}, R_{i}\right)=\alpha_{T}\left(T-T_{C}\right)+2\left(Q_{12}+Q_{11}\right) \times \\
& \times \frac{\Delta u}{s_{11}+s_{12}} \frac{2 R_{o} \Delta h}{\left(R_{o}+R_{i}\right) h}, \\
& \beta_{R}\left(R_{o}, R_{i}\right)= \\
& =\beta+2 \frac{\left(Q_{11}^{2}+Q_{12}^{2}\right) s_{11}-2 Q_{11} Q_{12} s_{12}}{s_{11}^{2}-s_{12}^{2}} \frac{2 R_{o} \Delta h}{\left(R_{o}+R_{i}\right) h} .
\end{aligned}
$$

Here, parameters $T_{\mathrm{C}}$ and $Q_{i j}$ are respectively the Curie temperature and electrostriction coefficient of the bulk material; $\alpha_{T}$ is proportional to the inverse Curie constant.

Note that under the condition $R_{o, i} \rightarrow \infty$ the renormalization given by Eqs. (3)-(4) coincides with the ones obtained for thin strained films by Pertsev et al. [30] allowing for stress relaxation [31], namely $\sigma(h) \approx \frac{\Delta u}{s_{11}+s_{12}} \frac{\Delta h}{h}$, here $\Delta h$ could be related with characteristic distance $h_{d}$ of stress relaxation and $\Delta u \rightarrow u_{m}$. In other words, we obtained that the stress relaxation occurs inside the shell. When $\Delta h \rightarrow 0$ the nanotube crystalline core appeared almost unstrained, i.e. $\sigma \approx 0$.

The surface part of the polarization-dependent free energy $G_{S}$ is thought to be proportional to square of polarization on the particle surface $S$, namely $G_{S}=\frac{\delta}{2} \int_{S} \frac{d s}{\lambda} P_{S}^{2} \quad(\lambda$ is the extrapolation length $[8,9])$.

The considered nanotube has upper and bottom surfaces $z=l / 2, z=-l / 2$ and sidewalls $\rho=R_{i}, \rho=R_{o}-\Delta h$, so its surface energy $G_{S}$ acquires the form:

$$
\begin{aligned}
& G_{S}=\delta \int_{0}^{2 \pi} d \psi\left(\int_{-l / 2}^{l / 2} d\left(\frac{R_{o}}{\lambda_{S}} P_{3}^{2}\left(\rho=R_{o}-\Delta h\right)+\frac{R_{i}}{\lambda_{S}} P_{3}^{2}\left(\rho=R_{i}\right)\right)+\right. \\
& \left.+\int_{R_{i}}^{R_{o}-\Delta h} \frac{\rho}{\lambda_{b}} d \rho\left(P_{3}^{2}\left(z=\frac{l}{2}\right)+P_{3}^{2}\left(z=-\frac{l}{2}\right)\right)\right) .
\end{aligned}
$$

We introduced longitudinal and lateral extrapolation lengths $\lambda_{b} \neq \lambda_{S}$. Hereinafter, we regard these extrapolation lengths positive.

Variation of the free energy expression $\delta G / \delta P_{3}=0$ yields the Euler-Lagrange equations with the boundary conditions on the tube faces $z= \pm l / 2$ and the sidewalls $\rho \approx R_{o, i}$. Under the presence of lattice pinning of viscous friction type, the polarization distribution should be found from Landau-Khalatnikov equation $\delta G / \delta P_{3}=-$ $\Gamma \partial P_{3} / \partial t$, where $\Gamma$ in kinetic coefficient. Along with the Poisson equation for the depolarization field $E_{3}^{d}=-\partial \varphi / \partial z$ they form the closed system:

$$
\begin{aligned}
& \int \Gamma \frac{\partial}{\partial t} P_{3}+\alpha_{R} P_{3}+\beta_{R} P_{3}^{3}+\gamma P_{3}^{5}- \\
& -\delta\left(\frac{\partial^{2}}{\partial z^{2}}+\frac{1}{\rho} \frac{\partial}{\partial \rho} \rho \frac{\partial}{\partial \rho}+\frac{1}{\rho^{2}} \frac{\partial^{2}}{\partial \psi^{2}}\right) P_{3}=E_{0}+E_{3}^{d}, \\
& \left.\left(P_{3}+\lambda_{b} \frac{d P_{3}}{d z}\right)\right|_{z=l / 2}=0, \\
& \left\{\left.\left(P_{3}-\lambda_{b} \frac{d P_{3}}{d z}\right)\right|_{z=-l / 2}=0,\right. \\
& \left.\left(P_{3}+\lambda_{S} \frac{d P_{3}}{d \rho}\right)\right|_{\rho=R_{o}}=0, \\
& \left|\left(P_{3}-\lambda_{S} \frac{d P_{3}}{d \rho}\right)\right|_{\rho=R_{i}}=0, \\
& \left\{\left(\frac{\partial^{2}}{\partial z^{2}}+\frac{1}{\rho} \frac{\partial}{\partial \rho} \rho \frac{\partial}{\partial \rho}+\frac{1}{\rho^{2}} \frac{\partial^{2}}{\partial \psi^{2}}\right) \varphi=4 \pi \operatorname{div} \mathbf{P},\right. \\
& \varphi\left(\rho=R_{o, e}\right)=0, \quad \varphi\left(z= \pm \frac{l}{2}\right)=0 .
\end{aligned}
$$

The polarization distribution in the ferroelectric phase should be found by direct variational methods allowing for possible polydomain states appearance in confined particles. At $\left(\lambda_{S} / R_{o}\right)<<1$ an exact series for the polarization and depolarization field distributions can be obtained [18]. The inequality $\left(\lambda_{S} / R_{o}\right)<<1$ is valid for typical extrapolation lengths $\lambda_{S}=0.3-5 \mathrm{~nm}$ and radii $R_{o}=30-500 \mathrm{~nm}$. Substituting the series for depolarization field and polarization into the free energy $G$ and integrating over nanoparticle volume, we obtained the free energy with renormalized coefficients for the average polarization. For infinite tubes and wires, the single-domain state is energetically preferable, since the depolarization field is absent and correlation energy is minimal for single-domain case. The depolarization field is highest for a single-domain nanotube, namely its upper estimation has the form:

$E_{3}^{d}(\rho, z)=-\frac{4 \pi}{1+\left(k_{01} l / 2 \pi R_{o}\right)^{2}} \times$

$\times\left(P_{3}(\rho, z)-\frac{2}{l} \int_{-l / 2}^{l / 2} d z P_{3}(\rho, z)\right)$.

Hereinafter, $k_{01}\left(R_{o}, R_{i}\right)$ is the lowest root of the equation $\quad J_{0}\left(k_{01} \frac{R_{i}}{R_{o}}\right) N_{0}\left(k_{01}\right)-J_{0}\left(k_{01}\right) N_{0}\left(k_{01} \frac{R_{i}}{R_{o}}\right)=0$ $\left(J_{0}(x)\right.$ and $N_{0}(x)$ are Bessel and Neiman functions of zero order, respectively). It should be noted that the depolarization field is absent outside the particles in the framework of our model. Therefore, the interaction of such nanoparticles is practically absent due to the screening. Their composite can be considered as the assembly of independent particles. 
For finite polydomain tubes, only numerical simulations have been performed. However, simple approximate analytical expression for the free energy renormalized coefficients has been obtained for the infinite single-domain tubes. We report the results below.

\section{Phase diagram of the long nanotubes}

We derived the interpolation for the paraelectricferroelectric transition temperature $T_{C R}\left(R_{o}, R_{i}\right)$ of the long nanotubes (for $l>>R_{o}$ depolarization field $E_{d} \rightarrow 0$ in accordance with Eq. (8)):

$$
\begin{aligned}
& T_{C R}\left(R_{o}, R_{i}\right)=T_{C}+\frac{2\left(Q_{11}+Q_{12}\right)}{\alpha_{T}} \sigma\left(R_{o}, R_{i}\right)- \\
& -\delta \frac{k_{01}^{2}\left(R_{o}, R_{i}\right)}{\alpha_{T} R_{o}^{2}} .
\end{aligned}
$$

An expression for the stress $\sigma\left(R_{o}, R_{i}\right)$ is given by Eqs (1). The first term in Eq. (9) is the bulk transition temperature, the second term is related to the coupling of stress with polarization via electrostriction effect, the third term is caused by correlation effects. The correlation term is always negative and thus only decreases the transition temperature, whereas the electrostriction term in Eq. (9) could be positive or negative depending on the sign of $\left(Q_{11}+Q_{12}\right) \sigma$. Note that both signs of $\left(Q_{11}+Q_{12}\right)$ are possible for different ferroelectrics, however $\left(Q_{11}+Q_{12}\right)>0$ for most of the perovskite ferroelectrics. Below, we demonstrate that increasing of transition temperature and thus ferroelectric properties conservation or even enhancement is possible, when the stress is compressive $(\sigma<0)$ and depolarization field is small enough.

Taking into account that the correlation radius at zero temperature is several (up to ten) lattice constants $\sqrt{\delta / \alpha_{T} T_{C}} \sim 1-4 \mathrm{~nm}$ (see, e.g., experimental data of Rodriguez et al. [32]) and using expression (1) for the stress, we introduced the parameters and dimensionless variables that correspond to the lattice constant units:

$$
\begin{aligned}
& R_{m}=\frac{2\left(Q_{11}+Q_{12}\right)}{\alpha_{T} T_{C}} \frac{\left(\vartheta_{i}^{c}-\vartheta_{i}^{s}\right) \Delta T-u_{g}}{s_{11}+s_{12}}, \\
& R_{\mu}=\frac{4\left(Q_{11}+Q_{12}\right)}{\alpha_{T} T_{C}} \frac{\mu}{R_{S}} \frac{s_{11}+2 s_{12}}{s_{11}+s_{12}}, \\
& R_{S}=\sqrt{\frac{\delta}{\alpha_{T} T_{C}}}, \quad r_{o}=\frac{R_{o}}{R_{S}}, \quad r_{i}=\frac{R_{i}}{R_{S}}, \\
& w=\frac{h}{R_{S}}, \quad \Delta w=\frac{\Delta h}{R_{S}} .
\end{aligned}
$$

Using approximate formula $k_{01}(x) \approx \pi /(1-x)-(1-x) /\left(4(1-x)^{2}+8 \pi x\right)$ for $x \rightarrow 1$, where $x=r_{i} / r_{o}$ one can write in dimensionless variables:

$$
\begin{aligned}
& T_{C R}\left(w, r_{i}, r_{o}\right) \approx T_{C}\left(1-\left(\frac{R_{\mu}}{r_{o}}+R_{m}\right) \times\right. \\
& \left.\times \frac{2 r_{o}}{r_{o}+r_{i}} \frac{\Delta w}{w}-\left(\frac{\pi^{2}}{w^{2}}-\frac{\pi / 2}{w^{2}+2 \pi r_{o} r_{i}}\right)\right),
\end{aligned}
$$

Let us make some estimations of the second and third terms in Eq. (11) for perovskites $\mathrm{BaTiO}_{3}$ and $\mathrm{Pb}(\mathrm{Zr}, \mathrm{Ti}) \mathrm{O}_{3}$. Using parameters $Q_{11}=0.11 \mathrm{~m}^{4} / \mathrm{C}^{2}, Q_{12}=-$ $0.043 \mathrm{~m}^{4} / \mathrm{C}^{2}, T_{\mathrm{C}}=400 \mathrm{~K}, \alpha_{T}=-7.4 \cdot 10^{-5} \mathrm{~K}^{-1}\left(\mathrm{BaTiO}_{3}\right)$ and $Q_{11}=0.089 \mathrm{~m}^{4} / \mathrm{C}^{2}, Q_{12}=-0.046 \mathrm{~m}^{4} / \mathrm{C}^{2}, T_{\mathrm{C}}=666 \mathrm{~K}$, $\alpha_{T}=-3 \cdot 10^{-5} \mathrm{~K}^{-1}\left(\mathrm{PbZr}_{0.5} \mathrm{Ti}_{0.5} \mathrm{O}_{3}\right) ; s_{11}=8 \cdot 10^{-12} \mathrm{~Pa}, s_{12}=-$ $2.5 \cdot 10^{-12} \mathrm{~Pa}, \Delta u \sim 5 \cdot\left(10^{-3}-10^{-2}\right)$ for por-Si or $\mathrm{Al}_{2} \mathrm{O}_{3}$ templates, $\mu=0.5-5 \mathrm{~N} / \mathrm{m}$ (see, e.g., Ref. [13]), we obtained that $R_{\mu} \sim 0.06-0.6,\left|R_{m}\right| \approx 0.5-5$ and $\Delta w \sim 0.2-$

2, respectively. Hence, both terms are comparable with unity and their contributions should be calculated carefully for the material under consideration.

The dependences of transition temperature $T_{C R}\left(w, r_{i}, r_{o}\right)$ on tube thickness $w$ for the cases of compressed $\left(R_{m}<0\right.$, solid curves), unstrained ( $\Delta w=0$, dashed curves) and tensiled ( $R_{m}>0$, dotted curves) tubes are compared in Fig. 2a. Corresponding spontaneous polarization $\quad P_{3}(T)=\sqrt{-\alpha_{R}(T) / \beta_{R}} \quad$ temperature dependences are presented in Fig. $2 \mathrm{~b}$. It is clear that nanotube compression leads to the maximum $T_{\max }>T_{\mathrm{C}}$ on transition temperature thickness dependence and to the corresponding enhancement of spontaneous polarization $P_{3}(T)>P_{S}(T)$ in the temperature range $T \approx T_{\max }$, whereas unstrained and tensiled nanotubes reveal monotonic dependences $T_{C R}\left(w, r_{i}, r_{o}\right)<T_{\mathrm{C}}$ with deteriorated polar properties $P_{3}(T)<P_{S}(T)$. Let us underline that the height and sharpness temperature maximum observed for compressed tubes decreases under the decrease of shell layer thicknesses $\Delta w$.

For the important case of thin tubes $\left(w<<r_{i}\right)$ one obtains from Eq. (11):

$T_{C R}(w) \approx T_{C}\left(1-R_{m} \frac{\Delta w}{w}-\frac{\pi^{2}}{w^{2}}\right)$.

Under the condition $R_{m}<0$, the second term tends to increase $T_{C R}$, while the third one always decreases it. At $R_{m}<0$, the competition between the contribution of strain effect represented by the second term and the correlation effect represented by the third one leads to the maximum appearance in $T_{C R}(w)$ dependence, namely:

$$
T_{\max }\left(w_{\max }\right) \approx T_{C}\left(1+\frac{R_{m}^{2} \Delta w^{2}}{4 \pi^{2}}\right), w_{\max } \approx-\frac{2 \pi^{2}}{R_{m} \Delta w} .
$$

For thin tubes, we also obtained simple analytical expressions for the critical radius at given temperature $T$ :

$w_{c r}^{ \pm}(T) \approx \frac{R_{m} \Delta w \pm \sqrt{4 \pi^{2}\left(1-T / T_{C}\right)+R_{m}^{2} \Delta w^{2}}}{2\left(1-T / T_{C}\right)}$. 

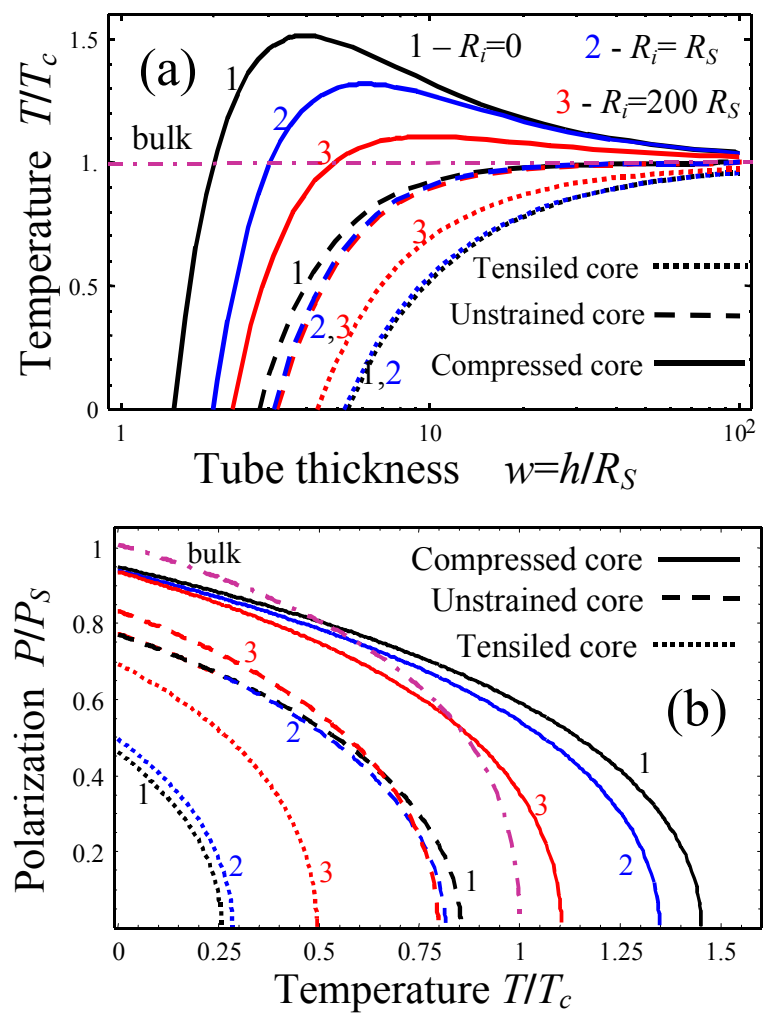

Fig. 2. (a) Transition temperature via tube thickness $w$ and (b) spontaneous polarization temperature dependence for tube thickness $w=7$ calculated from Eq. (11) for $R_{S}=4 \mathrm{~nm}$, $\Delta w=0.5, \quad R_{\mu}=0.5 \quad$ (which corresponds to PZT 50/50 parameters), $r_{i}=0 ; 1 ; 200$ (curves 1,2,3), $R_{m}=-4$ (solid curves), $R_{m}=0$ (dashed curves), $R_{m}=+4$ (dotted curves). Dashed-dotted curves correspond to the bulk material with spontaneous polarization $P_{S}(T)$.
Sign "+" before the radical in Eq. (14) corresponds to the both cases of unstrained, tensiled or compressed tubes (i.e., both signs of $R_{m}$ ), while both signs " \pm ” have sense for compressed tubes (i.e. two roots may exist at $R_{m}<0$ ) under the condition $T>T_{\mathrm{C}}$.

Finally let us discuss the influence of quartic term renormalization $\beta_{R}$ given by Eq. (4) on nanotubes phase diagrams in the particular case of the first order phase transitions in bulk material, i.e. when $\beta<0$. Since $\beta_{R}=\beta+\Delta \beta \frac{2 r_{o} \Delta w}{\left(r_{o}+r_{i}\right) w}$ in accordance with Eq. (4), the situation $\beta_{R}>0$ could appear at some tube thicknesses when $\Delta \beta>0$, i.e., the transition order in nanotube would be changed. This situation for $\mathrm{PbTiO}_{3}$ nanotubes phase diagrams in coordinates tube thickness-inner radius is demonstrated in Fig. 3 at room temperature and different stress signs $\left(R_{m}<0, R_{m}=0, R_{m}>0\right)$.

For the chosen material parameters, compressed tubes phase diagrams (a) have the thinnest region of paraelectric phase (PE) and widest region of the second order ferroelectric phase (FE-II) in comparison with unstrained (b) and tensiled (c) tubes.

\section{Piezoelectric force microscopy response of ferroelectric nanotubes}

Recently Morrison et al. [4, 5] demonstrated that long $\mathrm{Pb}(\mathrm{Zr}, \mathrm{Ti}) \mathrm{O}_{3}$ and $\mathrm{BaTiO}_{3}$ nanotubes possess perfect piezoelectric properties. For thin $\mathrm{PbZr}_{52} \mathrm{Ti}_{48} \mathrm{O}_{3}$ nanotube (outer diameter $700 \mathrm{~nm}$, wall thickness about $70 \mathrm{~nm}$, length $30 \mu \mathrm{m}$ ) they obtained rectangular hysteresis loop of effective piezoelectric response $d_{33}^{\text {eff }}(U)$. Poyato et al.

[17] with the help of PFM found that nanotube-patterned (a) Compressed

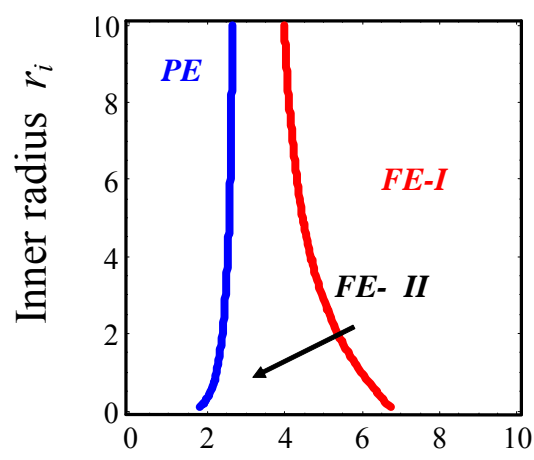

Tube thickness $w=h / R_{S}$ (b) Unstrained

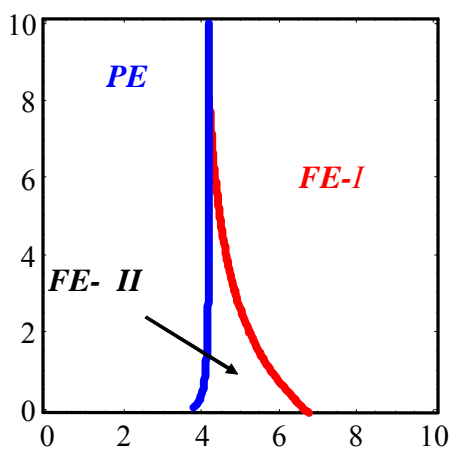

Tube thickness $w=h / R_{S}$ (c) Tensiled

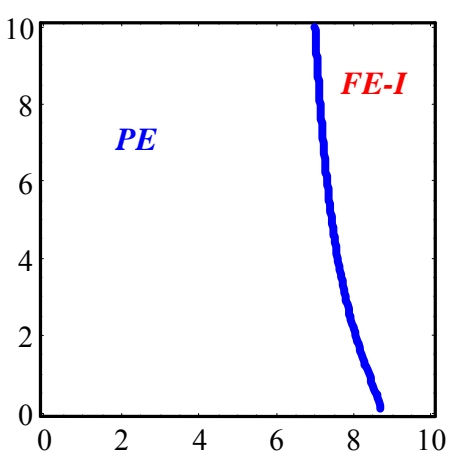

Tube thickness $w=h / R_{S}$

Fig. 3. Phase diagram in coordinates $\left\{r_{i}, w\right\}$ calculated at room temperature $T=297 \mathrm{~K}, R_{S}=4 \mathrm{~nm}, \Delta w=0.5, R_{\mu}=0.2, R_{m}=-4$ (a), $R_{m}=0$ (b), $R_{m}=+4$ (c). Material parameters correspond to $\mathrm{PbTiO}_{3} ; \mathrm{PE}$ - paraelectric phase, $\mathrm{FE}$ - ferroelectric phases of the first (I) and second (II) orders. 
$\mathrm{BaTiO}_{3}$ films of thickness 200-300 nm reveal piezoelectric hysteresis.

The effective vertical piezoresponse $d_{33}^{\text {eff }}$ is determined by the vertical mechanical displacement $u_{3}$ of ferroelectric sample surface caused by inhomogeneous electric field of a PFM probe biased under the voltage $U$, namely $d_{33}^{\text {eff }}(U)=u_{3} / U$ (see e.g. Refs. [33-35]). The local PFM response $d_{33}^{\text {eff }}$ is proportional to the stress piezoelectric tensor coefficients $d_{k l j}(\mathbf{r})$ representing ferroelectric material properties convoluted with the appropriate elastic Green function $G_{i j}(\mathbf{r}, \xi)$ [33-35]. Extending the results of Refs. [35, 37] for an empty tube embedded into non-piezoelectric matrix, we obtained that

$d_{33}^{\mathrm{eff}}(U)=\int_{-\infty}^{\infty} d \xi_{1} \int_{-\infty}^{\infty} d \xi_{2} \int_{0}^{\infty} d \xi_{3} \frac{\partial G_{i j}\left(x-\xi_{1}, y-\xi_{2}, \xi_{3}\right)}{\partial \xi_{k}} \times$

$\times E_{l}(\xi) \cdot c_{k j m n} d_{l n m}\left(y_{1}+\xi_{1}, y_{2}+\xi_{2}, \xi_{3}\right)$

$\approx t_{13}\left(R_{o}, R_{i}, a, \vartheta, v\right) d_{31}(U)+t_{51}\left(R_{o}, R_{i}, a, \vartheta\right) \times$

$\times d_{15}(U)+t_{33}\left(R_{o}, R_{i}, a, \vartheta\right) d_{33}(U)$.

The inhomogeneous electric field $E_{k}(\mathbf{r})=-\partial \varphi / \partial x_{k}$ is produced by the PFM tip inside of the sample, $c_{k j m n}$ are stiffness tensor components. Rather cumbersome integrals $t_{13}\left(R_{o}, R_{i}, a, \gamma, v\right)$ depend only on tube outer and inner radiuses, dielectric anisotropy coefficient $\vartheta=\sqrt{\varepsilon_{33} / \varepsilon_{11}}$, Poisson ratio $v$ and probe electric field distribution and tip position $a$ with respect to the tube (see Fig. 4a). In the approximation of effective point charge $Q$ simulating the probe electric field, the distance between the sample surface and the effective point charge is $d_{Q}=\varepsilon_{e} r_{0} / \sqrt{\varepsilon_{11} \varepsilon_{33}} \quad\left(r_{0}\right.$ is the probe apex curvature, $\varepsilon_{e}$ is ambient permittivity, $\left.d_{Q} \sim 10 \mathrm{~nm}\right)$. In accordance with Eq. (15), the effective piezoresponse $d_{33}^{\text {eff }}$ polarization dependence is fully determined by piezoelectric coefficients averaged over the piezoresponse volume. The conventional relationships between piezoelectric coefficients $d_{i j k}=2 \varepsilon_{0} Q_{j k l m}\left\langle\varepsilon_{i l} P_{m}\right\rangle$ in Voigth notation acquire explicit form:

$d_{33}=2 \varepsilon_{0} Q_{11}\left\langle\varepsilon_{33} P_{3}\right\rangle, \quad d_{31}=2 \varepsilon_{0} Q_{12}\left\langle\varepsilon_{33} P_{3}\right\rangle$,

$d_{15}=2 \varepsilon_{0} Q_{44}\left\langle\varepsilon_{11} P_{3}\right\rangle$.

$Q_{i j}$ is electrostriction tensor components in the Voigth notation.

In Table 1, we summarized calculated values of thermodynamic coercive field $E_{c}=2 \sqrt{-\alpha_{R}{ }^{3} / 27 \beta_{R}}$, spontaneous polarization $P_{S}$, dielectric permittivity $\varepsilon_{33}$ at room temperature, critical thickness $h_{\mathrm{cr}}$ and Curie temperature $T_{\mathrm{C}}$ for compressed $(\Delta u<0)$, unstrained $(\Delta u=0)$ and tensiled $(\Delta u>0)$ PZT (50/50) nanotubes (first 3 rows) in comparison with bulk material (last row). In the last columns of the table, we presented the values of electric field $E_{Q}(\rho, z) \cong U \frac{\left(\sqrt{\varepsilon_{11} / \varepsilon_{33}} z+d_{Q}\right) \sqrt{\varepsilon_{11} / \varepsilon_{33}} d_{Q}}{\left(\rho^{2}+\left(\sqrt{\varepsilon_{11} / \varepsilon_{33}} z+d_{Q}\right)^{2}\right)^{3 / 2}}$ produced by the PFM tip with parameter $d_{Q} \approx 25-50 \mathrm{~nm}$ on the tube surface $z=0$ and in the depth $z=d_{Q}$ for the coercive voltage $U_{c}^{ \pm} \approx 2.5 \mathrm{~V}$ measured experimentally. Material parameters used in calculations are given in Table 2 of Appendix A.

It is clear that obtained values of $E_{Q}(0,0)$ is in order of magnitude higher than thermodynamic coercive field $E_{c}$. The result is expectable, since in order to reverse the polarization inside the domain the PFM tip field should be higher than the coercive one not only just below the tip $(z=0, \rho=0)$ but also inside the layer of depth about $d_{Q}$ (typical penetration depth of piezoresponse) $[36,37]$. The values $E_{Q}\left(0, z=d_{Q}\right)$ actually appeared ten times smaller than $E_{Q}(0,0)$ and in a good agreement with calculated $E_{c}$ values.

We compare the piezoresponse loop shape obtained for $\mathrm{PbZr}_{52} \mathrm{Ti}_{48} \mathrm{O}_{3}$ nanotube [4] and $\mathrm{BaTiO}_{3}$ honeycomb [17] with our theoretical calculations in Figs. 4.

Table 1.

\begin{tabular}{|l|c|c|c|c|c|c|c|c|}
\hline \multicolumn{1}{c|}{$\begin{array}{c}\text { Tubes PZT } \\
(50 / 50)\end{array}$} & $\begin{array}{c}\text { Strain } \Delta u \\
(\%)\end{array}$ & $E_{c}(\mathrm{kV} / \mathrm{cm})$ & $P_{S}\left(\mu \mathrm{C} / \mathrm{cm}^{2}\right)$ & $\varepsilon_{33}$ & $\begin{array}{c}h_{\mathrm{cr}} \\
(\mathrm{nm})\end{array}$ & $T_{\mathrm{C}}(\mathrm{K})$ & $\begin{array}{c}E_{Q}(0,0) \\
(\mathrm{kV} / \mathrm{cm})\end{array}$ & $\begin{array}{c}E_{Q}\left(0, d_{Q}\right) \\
(\mathrm{kV} / \mathrm{cm})\end{array}$ \\
\hline \begin{tabular}{l} 
Sizes: \\
$R_{o}=700 \mathrm{~nm}$, \\
$h=70 \mathrm{~nm}$, \\
\cline { 2 - 9 }
\end{tabular} & -1 & 209 & 45 & 398 & 12.3 & 704 & $2060-1030$ & $219-110$ \\
\hline \multicolumn{2}{|l|}{1} & 128 & 39 & 583 & 23.0 & 586 & $1700-850$ & $232-116$ \\
\hline Bulk PZT (50/50) & 228 & 50 & 375 & - & 666 & $2100-1050$ & $214-107$ \\
\hline
\end{tabular}




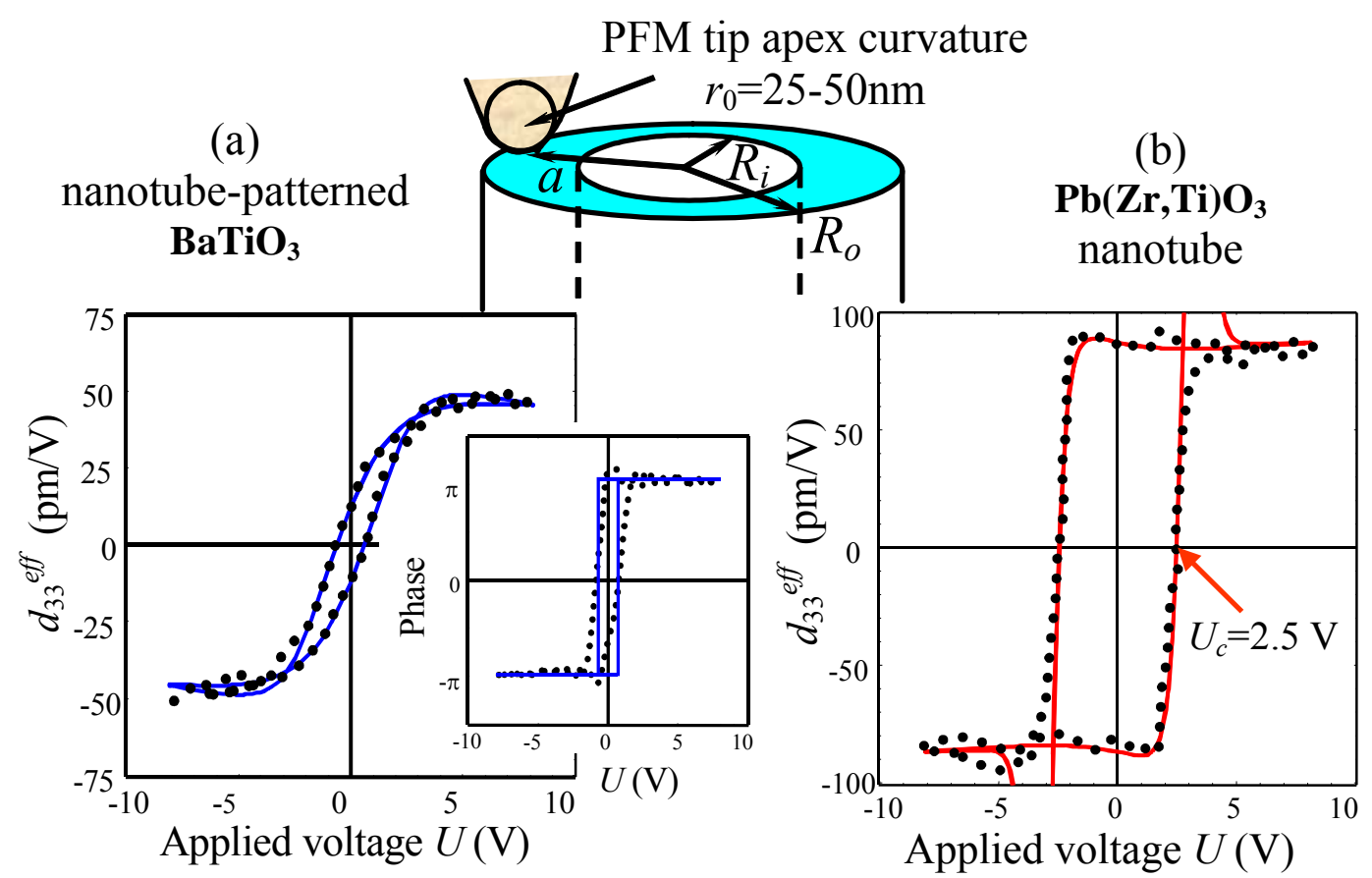

Fig. 4. (a) Effective piezoresponse $d_{33}^{\text {eff }}$ of $\mathrm{PbZr}_{52} \mathrm{Ti}_{48} \mathrm{O}_{3}$ nanotube (outer diameter $700 \mathrm{~nm}$, wall thickness $90 \mathrm{~nm}$, length about $30 \mu \mathrm{m}$ ) vs. applied voltage $U$. Squares are experimental data of Morrison et al. [4], solid curve is our fitting (13) for $R_{S} \approx 7, \Delta r=5, R_{\mu}=-5, \vartheta=0.25$ and PZT material parameters. (b) Effective piezoresponse $d_{33}^{\text {eff }}$ of nanotube-patterned $\mathrm{BaTiO}_{3}$ "honeycomb" (inner radius $50-100 \mathrm{~nm}$, film thickness about 200-300 nm) vs. applied voltage $U$. Squares are experimental data of Poyato et al. [17], solid curve is our fitting at $R_{i}=50 \mathrm{~nm}, R_{o}=62 \mathrm{~nm}, R_{S} \approx 6, \Delta r=5, R_{\mu}=-5$ and $\mathrm{BaTiO}_{3}$ material parameters. Dimensionless frequency of external field $\omega \Gamma /\left(\alpha_{T} T_{\mathrm{C}}\right)=0.15, \sqrt{\delta}=1 \mathrm{~nm}$.

\section{Conclusions}

- We reported ferroelectricity conservation and enhancement in long perovskite nanotubes compressed by their shell in contrast to ferroelectricity degradation in perovskite nanotubes tensiled by their shell. We demonstrated the stress-induced change of the ferroelectric phase transition order.

- The anisotropic stress as well as depolarization field decrease in long nanotubes are the keys to the ferroelectricity conservation. While the influence of depolarization field is obvious, the role of radial stress can be qualitatively understood as follows: although the radial stress conserves the inversion center, it leads to the shortrange forces strengthening in lateral direction (caused by the bond contraction) and their weakening in $z$-direction (caused by the bond elongation). As a result, the longrange correlations become more pronounced in polar direction in comparison with the short-range forces.
- We calculated the tube PFM response and demonstrated reasonable agreement with available experimental data.

\section{Acknowledgements}

Authors gratefully acknowledge the financial support from National Academy of Science of Ukraine, grant, joint Russian-Ukrainian grant NASU N 17-Ukr_a (RFBR N 08-02-90434), Ministry of Science and Education of Ukraine.

\section{Appendix A. Free energy with coefficients renormalized by elastic stresses}

Let us consider the nanotube with sidewalls covered with thin surface layers (shell). The free energy expansion on polarization $\mathbf{P}=\left(0,0, P_{3}\right)$ and stress $\sigma_{i j}$ powers has the form:

$$
G_{V}=\int_{-l / 2}^{l / 2} d z \int_{0}^{2 \pi} d \psi \int_{R_{i}}^{R_{o}} \rho d \rho\left(\begin{array}{l}
a_{1} P_{3}^{2}+a_{11} P_{3}^{4}+a_{111} P_{3}^{6}-Q_{11} \sigma_{33} P_{3}^{2}-Q_{12}\left(\sigma_{11}+\sigma_{22}\right) P_{3}^{2}- \\
-\left(\sigma_{11}+\sigma_{22}+\sigma_{33}\right) u_{t}-\frac{1}{2} s_{11}\left(\sigma_{11}^{2}+\sigma_{22}^{2}+\sigma_{33}^{2}\right)- \\
-s_{12}\left(\sigma_{11} \sigma_{22}+\sigma_{11} \sigma_{33}+\sigma_{33} \sigma_{22}\right)-\frac{1}{2} s_{44}\left(\sigma_{23}^{2}+\sigma_{13}^{2}+\sigma_{12}^{2}\right)
\end{array}\right)
$$


Subscripts 1, 2 and 3 denote Cartesian coordinates $x, y$ and $z$, respectively. Hereinafter, we use Voigt notation or matrix notation when it is necessary $(x x=1$, $y y=2, z z=3, z y=4, z x=5, x y=6$ ).

The internal isotropic strain $u_{i j}=u_{t} \delta_{i j}$, where $u_{t}=u_{t}^{c} \theta\left(R_{m}-\rho\right)+u_{t}^{s} \theta\left(\rho-R_{m}\right)$ is different in core and shell. Namely, $u_{t}^{s}=-2 \mu\left(s_{11}^{s}+2 s_{12}^{s}\right) / R_{o}+\vartheta_{i}^{s} \Delta T+u_{g}$ at $\rho \in\left(R_{i}, R_{m}\right)$ and $u_{t}^{c}=\vartheta_{i}^{c} \Delta T$ at $\rho \in\left(R_{m}, R_{o}\right)$. Here the interface radius $R_{m}=R_{o}-\Delta h$ is introduced. The temperature change after annealing is $\Delta T=T-T_{a}$, and $\vartheta_{i}^{s, c}$ are the linear temperature expansion coefficients. Polarization $P_{3}$ exists in the core region ("c") only, i.e. $P_{3}(\rho)=P_{3}(\rho) \theta\left(R_{m}-\rho\right)$. Hereinafter, superscripts "s" and "c" are related to the shell and core material, respectively. We will use them when necessary.

Minimization of the free energy (A.1) on stress components leads to the following equations of state:

$$
\left\{\begin{array}{c}
u_{11}=s_{11} \sigma_{11}+s_{12}\left(\sigma_{22}+\sigma_{33}\right)+Q_{12} P_{3}^{2}+u_{t}, \\
u_{22}=s_{11} \sigma_{22}+s_{12}\left(\sigma_{11}+\sigma_{33}\right)+Q_{12} P_{3}^{2}+u_{t}, \\
u_{33}=s_{11} \sigma_{33}+s_{12}\left(\sigma_{22}+\sigma_{11}\right)+Q_{11} P_{3}^{2}+u_{t}, \\
2 u_{23}=s_{44} \sigma_{23}, \quad 2 u_{13}=s_{44} \sigma_{13}, \quad 2 u_{12}=s_{44} \sigma_{12} .
\end{array}\right.
$$

Here $u_{i j}$ are the strain tensor components. Displacement vector components $u_{i}$ determine the strain tensor as $u_{i j}=\left(\partial u_{i} / \partial x_{j}+\partial u_{j} / \partial x_{i}\right) / 2$.

Distribution of mechanical displacement should satisfy the conditions of mechanical equilibrium $\partial \sigma_{i j} / \partial x_{i}=0$ as well as the appropriate boundary conditions $n_{i} \sigma_{i j}=0$ on the free surface and conditions of continuity at interfaces.

Let us introduce the cylindrical coordinates $(\rho, \psi, z)$ with $z$-axis coinciding with tube symmetry axis. Due to the axial symmetry of the system, only $\rho$ - and $z$ components of displacement are nonzero, $u_{\mathrm{\rho}}$ and $u_{z}$. In the general case, both of them may depend on $\rho$ and $z$, e.g. for the homogeneous thermal expansion $u_{\rho}=\rho \vartheta \Delta T, u_{z}=z \vartheta \Delta T$. At the first approximation, we suppose that both displacement components depend only on one coordinate, namely $u_{\rho}(\rho)$ and $u_{z}(z)$. Then, we try to find a general solution from the conditions of mechanical equilibrium that satisfies all the boundary conditions.

For the given ansatz, the deformation tensor nontrivial components are $u_{z z}=\partial u_{z} / \partial z, u_{\rho \rho}=\partial u_{\rho} / \partial \rho$ and $u_{\psi \psi}=u_{\rho} / \rho$. Shear components of stress and strain are zero. In this case, equations $\partial \sigma_{i j} / \partial x_{i}=0$ in the cylindrical coordinate system have the form:

$$
\left\{\begin{array}{l}
\frac{\partial \sigma_{z z}}{\partial z}=0, \\
\frac{\partial \sigma_{\psi \psi}}{\partial \rho}+\frac{\sigma_{\rho \rho}-\sigma_{\psi \psi}}{\rho}=0 .
\end{array}\right.
$$

Thus, equations of state (S.2) can be rewritten as follows:

$$
\left\{\begin{array}{l}
\sigma_{\rho \rho}=c_{11} \mathrm{v}_{\rho \rho}+c_{12}\left(\mathrm{v}_{\psi \psi}+\mathrm{v}_{z z}\right), \\
\sigma_{\psi \psi}=c_{11} \mathrm{v}_{\psi \psi}+c_{12}\left(\mathrm{v}_{\rho \rho}+\mathrm{v}_{z z}\right), \\
\sigma_{z z}=c_{11} \mathrm{v}_{z z}+c_{12}\left(\mathrm{v}_{\psi \psi}+\mathrm{v}_{\rho \rho}\right) .
\end{array}\right.
$$

Where $v_{\rho \rho}=u_{\rho \rho}-u_{t}-Q_{12} P_{3}^{2}$, $v_{\psi \psi}=u_{\psi \psi}-u_{t}-Q_{12} P_{3}^{2}, \quad v_{z z}=u_{z z}-u_{t}-Q_{11} P_{3}^{2} \quad$ and elastic stiffness $c_{11}=\left(s_{11}+s_{12}\right) /\left(\left(s_{11}-s_{12}\right)\left(s_{11}+2 s_{12}\right)\right)$, $c_{12}=-s_{12} /\left(\left(s_{11}-s_{12}\right)\left(s_{11}+2 s_{12}\right)\right)$ are introduced. After elementary transformations:

$$
\begin{aligned}
& \left\{\begin{array}{l}
u_{z}=a z, \quad u_{\rho}=b \rho+\frac{c}{\rho}, \\
u_{z z}=a, \quad u_{\rho \rho}=b-\frac{c}{\rho^{2}}, \quad u_{\psi \psi}=b+\frac{c}{\rho^{2}} .
\end{array}\right. \\
& \left\{\begin{array}{l}
\sigma_{\rho \rho}=a c_{12}+b\left(c_{11}+c_{12}\right)-\frac{c}{\rho^{2}}\left(c_{11}-c_{12}\right)- \\
-u_{t}\left(c_{11}+2 c_{12}\right)-\left(Q_{12}\left(c_{11}+c_{12}\right)+Q_{11} c_{12}\right) P_{3}^{2}, \\
\sigma_{\psi \psi}=a c_{12}+b\left(c_{11}+c_{12}\right)+\frac{c}{\rho^{2}}\left(c_{11}-c_{12}\right)- \\
-u_{t}\left(c_{11}+2 c_{12}\right)-\left(Q_{12}\left(c_{11}+c_{12}\right)+Q_{11} c_{12}\right) P_{3}^{2}, \\
\sigma_{z z}=a c_{11}+2 b c_{12}-u_{t}\left(c_{11}+2 c_{12}\right)- \\
-\left(Q_{11} c_{11}+2 Q_{12} c_{12}\right) P_{3}^{2} .
\end{array}\right.
\end{aligned}
$$

The boundary conditions of mechanical equilibrium $n_{i} \sigma_{i j}=0$ on the external surfaces of a cylindrical solid body have the following form:

$$
\left\{\begin{array}{l}
\sigma_{\rho \rho}^{s}\left(\rho=R_{o}\right)=0, \quad \sigma_{\rho \rho}^{c}\left(\rho=R_{i}\right)=0 \\
\sigma_{z z}^{c}\left(z= \pm l / 2, \rho \in\left(R_{i}, R_{m}\right)\right)=0 \\
\sigma_{z z}^{s}\left(z= \pm l / 2, \rho \in\left(R_{m}, R_{o}\right)\right)=0
\end{array}\right.
$$

On the interface between two different mediums $\left(\rho=R_{m}\right)$, continuity conditions of stress tensor normal components and the displacement vector should be satisfied:

$$
\left\{\begin{array}{l}
\sigma_{\rho \rho}^{s}\left(\rho=R_{m}+0\right)=\sigma_{\rho \rho}^{c}\left(\rho=R_{m}-0\right), \\
u_{\rho}\left(\rho=R_{m}+0\right)=u_{\rho}\left(\rho=R_{m}-0\right), \\
u_{z}\left(\rho=R_{m}+0\right)=u_{z}\left(\rho=R_{m}-0\right) .
\end{array}\right.
$$


Therefore, for the tube consisting of two layers, we have seven boundaries and interfacial conditions (A.48). At the same time, we have a general solution (A.3) for each layer, depending on the independent constants $\left(\left\{a^{c}, b^{c}, c^{c}\right\}\right.$ and $\left.\left\{a^{s}, b^{s}, c^{s}\right\}\right)$ in each layer. For two layers, we have six constants, which do not allow us to fulfill seven independent conditions simultaneously. Since we are interested in the solution for a long tube, we have to satisfy the conditions on the lateral surfaces. As for the conditions on faces at $z= \pm l / 2$, the natural way to solve this problem approximately is to nullify the overall force acting on the face of the tube, i.e. $\int \sigma_{z z}(z= \pm l / 2, \rho) \rho d \rho=0$ and obtain the solution in $R_{o}<\rho<R_{i}$

the sense of the Saint Venant principle.

Under the conditions $\Delta h<<h$ and $P_{3}^{2}(l / 2, \rho)=P_{3}^{2}(-l / 2, \rho)$, we finally obtain the solution inside the core and shell regions:

$$
\begin{aligned}
& \sigma_{11}^{c}+\sigma_{22}^{c}=\sigma_{\rho \rho}^{c}+\sigma_{\psi \psi}^{c}=-\frac{1}{s_{11}^{s}+s_{12}^{s}} \times \\
& \times \frac{2 R_{o} \Delta h}{\left(R_{o}+R_{i}\right) h}\left[\Delta u+\frac{Q_{12} s_{11}^{s}-Q_{11} s_{12}^{s}}{s_{11}^{s}-s_{12}^{s}} P_{3}^{2}\right], \\
& \sigma_{33}^{c}=\sigma_{z z}^{c}=-\frac{1}{s_{11}^{s}+s_{12}^{s}} \times \\
& \times \frac{2 R_{o} \Delta h}{\left(R_{o}+R_{i}\right) h}\left[\Delta u+\frac{Q_{11} s_{11}^{s}-Q_{12} s_{12}^{s}}{s_{11}^{s}-s_{12}^{s}} P_{3}^{2}\right] \text {, } \\
& \sigma_{z z}^{s}=\frac{1}{s_{11}^{s}+s_{12}^{s}}\left[\Delta u+\frac{Q_{11} s_{11}^{s}-Q_{12} s_{12}^{s}}{s_{11}^{s}-s_{12}^{s}} \times\right. \\
& \left.\times \int_{R_{m}<\rho<R_{i}} \frac{2 \rho d \rho}{R_{m}^{2}-R_{i}^{2}} P_{3}^{2}(l / 2, \rho)\right], \\
& \sigma_{\rho \rho}^{s}+\sigma_{\psi \psi}^{s}=\frac{1}{s_{11}^{s}+s_{12}^{s}}\left[\Delta u+\frac{Q_{12} s_{11}^{s}-Q_{11} s_{12}^{s}}{s_{11}^{s}-s_{12}^{s}} \times\right. \\
& \left.\times \int_{R_{m}<\rho<R_{i}} \frac{2 \rho d \rho}{R_{m}^{2}-R_{i}^{2}} P_{3}^{2}(l / 2, \rho)\right], \\
& \sigma_{\rho \psi}=0, \quad \sigma_{\rho z}=0, \quad \sigma_{z \psi}=0 \text {. }
\end{aligned}
$$

$\Delta u=u_{t}^{c}-u_{t}^{s}=\frac{2 \mu}{R_{o}}\left(s_{11}^{s}+2 s_{12}^{s}\right)+\left(\vartheta_{i}^{c}-\vartheta_{i}^{s}\right) \Delta T-u_{g}$

difference

introduced. Let us recap that superscript "s" is related to the shell material, "c" is related to the core. Hereinafter, we suppose that $S_{i j}^{s} \approx s_{i j}^{c} \approx s_{i j}$. Invariants:

$$
\begin{aligned}
& \operatorname{Sp}\left(\sigma_{i i}^{c}\right)=-\frac{1}{S_{11}+S_{12}} \times \\
& \times \frac{2 R_{o} \Delta h}{\left(R_{o}+R_{i}\right) h}\left(2 \Delta u+\left(Q_{12}+Q_{11}\right) P_{3}^{2}\right),
\end{aligned}
$$

$$
\begin{aligned}
& S p\left(\sigma_{i i}^{s}\right)=\frac{1}{s_{11}+s_{12}}\left(2 \Delta u+\left(Q_{12}+Q_{11}\right) \times\right. \\
& \left.\times \int_{R_{m}<\rho<R_{i}} \frac{2 \rho d \rho}{R_{m}^{2}-R_{i}^{2}} P_{3}^{2}(l / 2, \rho)\right), \\
& \sigma_{i i}^{s^{2}} \approx \frac{1}{\left(s_{11}+s_{12}\right)^{2}}\left[\Delta u^{2}+2 \Delta u\left(Q_{11}+Q_{12}\right) \times\right. \\
& \times \int_{R_{m}<\rho<R_{i}} \frac{2 \rho d \rho}{R_{m}^{2}-R_{i}^{2}} P_{3}^{2}(l / 2, \rho)+ \\
& +\frac{\left(Q_{11}^{2}+Q_{11}^{2}\right)\left(s_{11}^{2}+s_{12}^{2}\right)-4 Q_{11} Q_{12} s_{11} S_{12}}{\left(s_{11}-s_{12}\right)^{2}} \times \\
& \left.\times\left(\int_{R_{m}<\rho<R_{i}} \frac{2 \rho d \rho}{R_{m}^{2}-R_{i}^{2}} P_{3}^{2}(l / 2, \rho)\right)^{2}\right] .
\end{aligned}
$$

Using (A.10) and the condition $\Delta h<<h$, the polarization dependent part of the core free energy expansion (A.1) acquires the form:

$$
\begin{aligned}
& G_{V} \approx \int_{-l / 2}^{l / 2} d z \int_{0}^{2 \pi} d \psi \int_{R_{i}}^{R_{o}-\Delta h} \rho d \rho \times \\
& \times\left(\begin{array}{l}
\left(a_{1}+\left(Q_{11}+Q_{12}\right) \frac{\Delta u}{s_{11}+s_{12}} \frac{2 R_{o} \Delta h}{\left(R_{o}+R_{i}\right) h}\right) P_{3}^{2}+a_{111} P_{3}^{6}+ \\
+\left(a_{11}+\frac{2 R_{o} \Delta h}{\left(R_{o}+R_{i}\right) h} \frac{\left(Q_{11}^{2}+Q_{12}^{2}\right) s_{11}-2 Q_{11} Q_{12} s_{12}}{2\left(s_{11}^{2}-s_{12}^{2}\right)}\right) P_{3}^{4}
\end{array}\right) .
\end{aligned}
$$

In Eq. (A.11) we neglected the terms proportional $(\Delta h / h)^{2}$.

Table 2.

\begin{tabular}{|l|c|}
\hline Parameters of single crystals & PZT 50/50 \\
\hline$T_{\mathrm{C}}(\mathrm{K})$ & 666 \\
\hline$\alpha_{T}\left(10^{5} \mathrm{~m} \mathrm{~F}^{-1} \mathrm{~K}^{-1}\right)$ & 2.66 \\
\hline$\beta\left(10^{8} \mathrm{~m}^{5} \mathrm{~F}^{-1} \mathrm{C}^{-2}\right)$ & 1.898 \\
\hline$\gamma\left(10^{8} \mathrm{~m}^{9} \mathrm{~F}^{-1} \mathrm{C}^{-4}\right)$ & 8.016 \\
\hline$Q_{11}\left(\mathrm{~m}^{4} \mathrm{C}^{-2}\right)$ & 0.097 \\
\hline$Q_{12}\left(\mathrm{~m}^{4} \mathrm{C}^{-2}\right)$ & -0.046 \\
\hline$s_{11}\left(10^{-12} \mathrm{~m}^{2} \mathrm{~N}^{-1}\right)$ & 10.5 \\
\hline$s_{12}\left(10^{-12} \mathrm{~m}^{2} \mathrm{~N}^{-1}\right)$ & -3.7 \\
\hline$\varepsilon_{11} ; \varepsilon_{33}$ & $1700 ; 730$ \\
\hline
\end{tabular}

PZT bulk values $d_{31}=-93.5 \mathrm{pm} / \mathrm{V}, d_{15}=494 \mathrm{pm} / \mathrm{V}, d_{33}=$ $220 \mathrm{pm} / \mathrm{V}, \varepsilon_{11}=1180, \varepsilon_{33}=730$. BTO bulk values $d_{13}=-$ $34.5 \mathrm{pm} / \mathrm{V}, d_{33}=86 \mathrm{pm} / \mathrm{V}, d_{15}=392 \mathrm{pm} / \mathrm{V}, \varepsilon_{11}=2920$, $\varepsilon_{33}=168$.

\section{References}


1. D. Yadlovker and S. Berger, Uniform orientation and size of ferroelectric domains // Phys. Rev. B 71, 184112 (2005).

2. G. Geneste, E. Bousquest, J. Junquera, and P. Chosez, Finite-size effects in $\mathrm{BaTiO}_{3}$ nanowires // Appl. Phys. Lett. 88, 112906 (2006).

3. Y. Luo, I. Szafraniak, N.D. Zakharov, V. Nagarajan, M. Steinhart, R.B. Wehrspohn, J.H. Wendroff, R. Ramesh, M. Alexe, Nanoshell tubes of ferroelectric lead zirconate titanate and barium titanate // Appl. Phys. Lett. 83, p. 440 (2003).

4. D. Morrison, L. Ramsay, and J.F. Scott, High aspect ratio piezoelectric strontium-bismuthtantalate nanotubes // J. Phys.: Condens. Matter 15, p. L527 (2003).

5. F.D. Morrison Y. Luo, I. Szafraniak, et al., Ferroelectric nanotubes // Rev. Adv. Mater. Sci. 4, p. 114 (2003).

6. M.E. Lines and A.M. Glass, Principles and Applications of Ferroelectrics and Related Phenomena. Clarendon Press, Oxford, 1977.

7. L.D. Landau and E.M. Lifshits, Electrodynamics of Continuous Media. Butterworth Heinemann, Oxford, 1980.

8. D.R. Tilley, Finite-size effects on phase transitions in ferroelectrics, In: Ferroelectric Thin Films, ed. C. Paz de Araujo, J.F. Scott, and G.W. Teylor. Gordon and Breach, Amsterdam, 1996.

9. C.L. Wang and S.R.P. Smith, Landau theory of the size-driven phase transition in ferroelectrics // J. Phys.: Condens. Matter 7, p. 7163 (1995).

10. I. Rychetsky and O. Hudak, The ferroelectric phase transition in small spherical particles // J. Phys.: Condens. Matter 9, p. 4955 (1997).

11. D.D. Fong, G.B. Stephenson, S.K. Streiffer, J.A. Eastman, O. Auciello, P.H. Fuoss, and C. Thompson, Ferroelectricity in ultrathin perovskite films // Science 304, p. 1650 (2004).

12. S.K. Mishra and D. Pandey, Effect of particle size on the ferroelectric behaviour of tetragonal and rhombohedral $\mathrm{Pb}\left(\mathrm{Zr}_{\mathrm{x}} \mathrm{Ti}_{1-\mathrm{x}}\right) \mathrm{O}_{3}$ ceramics and powders // J. Phys.: Condens. Matter 7, p. 9287 (1995).

13. K. Uchino, E. Sadanaga, and T. Hirose, Dependence of the crystal structure on particle size in barium titanate // J. Amer. Ceram. Soc. 72, p. 1555 (1989).

14. M.D. Glinchuk and A.N. Morozovska, Effect of surface tension and depolarization field on ferroelectric nanomaterials properties // Phys. status solidi (b) 238, p. 81 (2003).

15. H. Huang, C.Q. Sun, Zh. Tianshu and P. Hing, Grain-size effect on ferroelectric $\mathrm{Pb}\left(\mathrm{Zr}_{1-\mathrm{x}} \mathrm{Ti}_{\mathrm{x}}\right) \mathrm{O}_{3}$ solid solutions induced by surface bond contraction // Phys. Rev. B 63, 184112 (2001).

16. E.D. Mishina, K.A. Vorotilov, V.A. Vasil'ev, A.S. Sigov, N. Ohta, and S. Nakabayashi, Porous silicon-based ferroelectric nanostructures //
Zhurnal. Experim. Teor. Fiziki 95 (3), p. 502-504 (2002) (in Russian).

17. R. Poyato and B.D. Huey and N.P. Padture, Local piezoelectric and ferroelectric responses in nanotube-patterned thin films of $\mathrm{BaTiO} 3$ synthesized hydrothermally at $200{ }^{\circ} \mathrm{C} / / \mathrm{J}$. Mater. Res. 21, 547 (2006).

18. A.N. Morozovska, E.A. Eliseev, and M.D. Glinchuk, Ferroelectricity enhancement in confined nanorods: Direct variational method // Phys. Rev. B 73, 214106 (2006).

19. A.N. Morozovska, E.A. Eliseev, and M.D. Glinchuk, Size effects and depolarization field influence on the phase diagrams of cylindrical ferroelectric nanoparticles // Physica B 387, p. 358 (2007).

20. A.N. Morozovska, M.D. Glinchuk, and E.A. Eliseev, Ferroelectricity enhancement in ferroelectric nanotubes // Phase Transitions 80, No. 1-2, p. 71-77 (2007).

21. J. Freund, J. Halbritter, and J.K.H. Horber, How dry are dried samples? Water adsorption measured by STM // Microsc. Res. Tech. 44, p. 327-338 (1999).

22. R. Kretschmer and K. Binder, Surface effects on phase transition in ferroelectrics and dipolar magnets // Phys. Rev. B 20, p. 1065 (1976).

23. J.C. Niepce, Permittivity of fine grained $\mathrm{BaTiO}_{3} / /$ Electroceramics 4, No. 5-7, p. 29 (1994).

24. P. Perriat, J.C. Niepce, G. Gaboche, Thermodynamic consideration of the grain size dependence of materials properties // J. Thermal Analysis 41, p. 635-649 (1994).

25. M.D. Glinchuk, A.N. Morozovska, Radiospectroscopy and dielectric properties of nanomaterials // Fizika tverdogo tela 45 (8), p. 1510-1518 (2003) (in Russian).

26. V.A. Shchukin and D. Bimberg, Spontaneous ordering of nanostructures on crystal surfaces // Reviews of Modern Physics 71 (4), p. 1125-1171 (1999).

27. S.V. Kalinin, E.A. Eliseev, and A.N. Morozovska, Materials contrast in piezoresponse force microscopy // Appl. Phys. Lett. 88, p. 232904-1-3 (2006).

28. A.N. Morozovska, S.V. Svechnikov, The influence of size effects on thin films local piezoelectric response // Semiconductor Physics, Quantum Electronics \& Optoelectronics 10 (4), p. 36-41 (2007).

29. L.D. Landau and E.M. Lifshitz, Theory of Elasticity. Theoretical Physics, Vol. 7. Butterworth-Heinemann, Oxford, U.K., 1998.

30. N.A. Pertsev, A.G. Zembilgotov, and A.K. Tagantsev, Effect of mechanical boundary conditions on phase diagrams of epitaxial ferroelectric thin films // Phys. Rev. Lett. 80 (9), p. 1988-1991 (1998). 
31. J.S. Speck, and W. Pompe, Domain configurations due to multiple misfit relaxation mechanisms in epitaxial ferroelectric thin films. I. Theory // $J$. Appl. Phys. 76 (1), p. $466-476$ (1994).

32. B.J. Rodriguez, S. Jesse, A.P. Baddorf, and S.V. Kalinin, High resolution electromechanical imaging of ferroelectric materials in a liquid environment by piezoresponse force Microscopy // Phys. Rev. Lett. 96 (23), 237602 (2006).

33. A.N. Morozovska, S.V. Svechnikov, E.A. Eliseev, and S.V. Kalinin, Extrinsic size effect in piezoresponse force microscopy of thin films // Phys. Rev. B 76 (5), 054123-1-5 (2007).

34. A.N. Morozovska, E.A. Eliseev, G.S. Svechnikov, V. Gopalan, and S.V. Kalinin, Effect of the intrinsic width on the piezoelectric force microscopy of a single ferroelectric domain wall // J. Appl. Phys. 103 (12), 124110-1-8 (2008).
35. A.N. Morozovska, E.A. Eliseev, S.L. Bravina, and S.V. Kalinin, Resolution function theory in piezoresponse force microscopy: domain wall profile, spatial resolution, and tip calibration // Phys. Rev. B 75 (17), 174109-1-18 (2007).

36. A.N. Morozovska, S.V. Svechnikov, E.A. Eliseev, S. Jesse, B.J. Rodriguez, S.V. Kalinin, Piezoresponse Force Spectroscopy of FerroelectricSemiconductor Materials // J. Appl. Phys. 102 (11), 114108-1-14 (2007).

37. A.N. Morozovska, S.V. Kalinin, E.A. Eliseev, V. Gopalan, and S.V. Svechnikov, The interaction of an 180-degree ferroelectric domain wall with a biased scanning probe microscopy tip: effective wall geometry and thermodynamics in GinzburgLandau-Devonshire theory // Phys. Rev. B 78 (12), 125407-1-11 (2008). 